\title{
EXTREME PRESERVERS OF MAXIMAL COLUMN RANK INEQUALITIES OF MATRIX MULTIPLICATIONS OVER SEMIRINGS
}

\author{
Seok-Zun Song, Kwon-Ryong Park, and L. Hernandez Encinas
}

\begin{abstract}
We characterize linear operators that preserve sets of matrix ordered pairs which satisfy extreme cases with respect to maximal column rank inequalities of matrix multiplications over semirings.
\end{abstract}

\section{Introduction}

One of the most active and fertile subjects in matrix theory is the study of those linear operators on matrices that leave certain properties or relations of matrices invariant. For a field $F, M_{n}(F)$ be the vector space of all $n \times n$ matrices over $F$. A lot of work has been done on the problems of determining the linear operators on $M_{n}(F)$ that leave certain matrix subsets or matrix properties invariant. For a survey of these types of problems, see [5]. Although the linear preservers concerned are mostly linear operators on matrix spaces over fields or rings, the same problem has been extended to matrices over various semirings.

Recently, Beasley and his colleagues investigated rank inequalities of matrices over semirings ([1]) and characterized the linear operators that preserve extreme set of matrix pairs for rank inequality cases ([2] and [3]). This research extends the linear preserver problems to the set of matrix pairs from the set of single matrices. These characterization problems are open even over fields as well as over semirings ([5]). The structure of matrix varieties which arise as extremal cases in the inequalities is not known over fields and over semirings. A usual way to generate elements of such a variety is to find a matrix pairs which belongs to it and to act on this set by various linear operators that preserve this variety. Song and his colleagues characterized the linear operators that preserve maximal column $\operatorname{rank}([4]$ and $[6])$.

Received February 19, 2008; Revised January 19, 2009.

2000 Mathematics Subject Classification. Primary 15A03, 15A04, 15A45.

Key words and phrases. linear operator, rank inequality, maximal column rank.

This research was supported by Basic Science Research Program through the National Research Foundation of Korea(NRF) funded by the Ministry of Education, Science and Technology (No. 2009-0073164). 
In this paper, we characterize the linear operators that preserve the extreme sets of matrix pairs which satisfy multiplicative properties with respect to maximal column rank of matrices over semirings.

\section{Preliminaries and definitions}

Definition 2.1. A semiring $\mathbb{S}$ consists of a set and two binary operations, addition and multiplication, such that:

- $\mathbb{S}$ is an Abelian monoid under addition (identity denoted by 0 );

- $\mathbb{S}$ is a semigroup under multiplication (identity, if any, denoted by 1 );

- multiplication is distributive over addition on both sides;

- $s 0=0 s=0$ for all $s \in \mathbb{S}$.

In this paper we will always assume that there is a multiplicative identity 1 in $\mathbb{S}$ which is different from 0 .

In particular, a semiring $\mathbb{S}$ is called antinegative if the zero element is the only element with an additive inverse.

Throughout this paper, we will assume that all semirings are antinegative and have no zero divisors.

Definition 2.2. The binary Boolean algebra consists of the set $\mathbb{B}=\{0,1\}$ equipped with two binary operations, addition and multiplication. The operations are defined as usual except that $1+1=1$.

Let $\mathbb{M}_{m, n}(\mathbb{S})$ denote the set of $m \times n$ matrices with entries from the semiring $\mathbb{S}$. If $m=n$, we use the notation $\mathbb{M}_{n}(\mathbb{S})$ instead of $\mathbb{M}_{n, n}(\mathbb{S})$. The matrix $I_{n}$ is the $n \times n$ identity matrix, $J_{m, n}$ is the $m \times n$ matrix of all ones, $O_{m, n}$ is the $m \times n$ zero matrix. We omit the subscripts when the order is obvious from the context and we write $I, J$, and $O$, respectively. Let $R_{i}$ denote the matrix whose $i^{\text {th }}$ row is all ones and all other rows are zero, and $C_{j}$ denote the matrix whose $j^{\text {th }}$ column is all ones and all other columns are zero.

The matrix $E_{i, j}$, called a cell, denotes the matrix with 1 in $(i, j)$ position and zero elsewhere. A weighted cell is any nonzero scalar multiple of a cell, that is, $\alpha E_{i, j}$ is a weighted cell for any $0 \neq \alpha \in \mathbb{S}$.

For a matrix $A, A^{t}$ denotes the transpose of $A$. A line of a matrix $A$ is a row or a column of $A$. We let $\mathbb{Z}(\mathbb{S})$ denote the center of the semiring $\mathbb{S}$, and $|A|$ denote the number of nonzero entries in the matrix $A$, and $A\left[i_{1}, \ldots, i_{k} \mid j_{1}, \ldots, j_{l}\right]$ denote the $k \times l$-submatrix of $A$ which lies in the intersection of the $i_{1}, \ldots, i_{k}$ rows and $j_{1}, \ldots, j_{l}$ columns.

Let $\Delta_{m, n}=\{(i, j) \mid i=1, \ldots, m ; j=1, \ldots, n\}$. If $m=n$, we use the notation $\Delta_{n}$ instead of $\Delta_{n, n}$.

Definition 2.3. An element in $\mathbb{M}_{n, 1}(\mathbb{S})$ is called a vector over $\mathbb{S}$.

A set of vectors with entries from a semiring is called linearly independent if there is no vector in this set that can be expressed as a nontrivial linear combination of the others. 
The matrix $A \in \mathbb{M}_{m, n}(\mathbb{S})$ is said to be of maximal column rank $k(m c(A)=$ $k$ ) if $k$ is the maximal number of the columns of $A$ which are linearly independent.

The matrix $A \in \mathbb{M}_{m, n}(\mathbb{S})$ is said to be of maximal row rank $k(m r(A)=k)$ if $k$ is the maximal number of the rows of $A$ which are linearly independent.

The matrix $A \in \mathbb{M}_{m, n}(\mathbb{S})$ is said to be of factor $\operatorname{rank} k(\operatorname{rank}(A)=k)$ if there exist matrices $B \in \mathbb{M}_{m, k}(\mathbb{S})$ and $C \in \mathbb{M}_{k, n}(\mathbb{S})$ such that $A=B C$ and $k$ is the smallest positive integer for which such factorization exists. By definition, the only matrix with factor rank 0 is the zero matrix, $O$.

Remark 2.4. It follows that

$$
1 \leq \operatorname{rank}(A) \leq m c(A) \leq n
$$

for all nonzero matrix $A \in \mathbb{M}_{m, n}(\mathbb{S})$.

If $\mathbb{S}$ is a subsemiring of a real field, then there is a real rank function $\rho(A)$ for any matrix $A \in \mathbb{M}_{m, n}(\mathbb{S})$, which is considered as a matrix over real field. Easy examples show that over semirings these functions are not equal in general. However, the inequality $m c(A) \geq \rho(A)$ always holds.

The behavior of the real rank function $\rho$ with respect to matrix multiplication and addition is given by well-known Frobenius, Schwartz and Sylvester inequalities. Arithmetic properties of maximal row and maximal column ranks depend on the structure of semiring of entries.

Theorem 2.5 ([1]). Let $\mathbb{S}$ be an antinegative semiring without zero divisors and let $A \in \mathbb{M}_{m, n}(\mathbb{S}), B \in \mathbb{M}_{n, k}(\mathbb{S})$ with $A \neq O, B \neq O$. Then

1. $m c(A B) \leq m c(B)$.

If $m c(A)+m c\left(B^{t}\right)>n$, then

2. $m c(A B) \geq 1$.

For $A \in \mathbb{M}_{m, n}(\mathbb{S})$ and $B \in \mathbb{M}_{n, k}(\mathbb{S})$, one has that

3. $m c(A B) \geq 0$.

4. $m c(A B) \geq \rho(A)+\rho(B)-n$.

As was proved in [1], these inequalities are sharp and the best possible.

The following example shows that standard analogs for upper bound of the factor rank of product of two matrices do not hold for maximal column rank, that is, $\min \{m c(A), m c(B)\}$ is not greater than $m c(A B)$.

Example 2.6. Let $A=(3,7,7) \in \mathbb{M}_{1,3}\left(\mathbb{Z}^{+}\right), B=\left[\begin{array}{lll}1 & 1 & 1 \\ 0 & 1 & 1 \\ 0 & 0 & 1\end{array}\right] \in \mathbb{M}_{3}\left(\mathbb{Z}^{+}\right)$, where $\mathbb{Z}^{+}$is the semiring of nonnegative integers. Then $m c(A)=2, m c(B)=3$, and $m c(A B)=m c(3,10,17)=3$ over $\mathbb{Z}^{+}$.

Definition 2.7. For matrices $X=\left[x_{i, j}\right]$ and $Y=\left[y_{i, j}\right]$ in $\mathbb{M}_{m, n}(\mathbb{S})$, the matrix $X \circ Y$ denotes the Hadamard or Schur product, i.e., the $(i, j)^{\text {th }}$ entry of $X \circ Y$ is $x_{i, j} y_{i, j}$.

We say that the matrix $A$ dominates the matrix $B$ if and only if $b_{i, j} \neq 0$ implies that $a_{i, j} \neq 0$, and we write $A \geq B$ or $B \leq A$ in this case. 
Definition 2.8. Let $\mathbb{S}$ be a semiring, not necessarily commutative. An operator $T: \mathbb{M}_{m, n}(\mathbb{S}) \rightarrow \mathbb{M}_{m, n}(\mathbb{S})$ is called linear if $T(\alpha X)=\alpha T(X), T(X \beta)=$ $T(X) \beta$, and $T(X+Y)=T(X)+T(Y)$ for all $X, Y \in \mathbb{M}_{m, n}(\mathbb{S}), \alpha, \beta \in \mathbb{S}$.

We say that an operator $T$ preserves a set $\mathbb{P}$ if $X \in \mathbb{P}$ implies that $T(X) \in \mathbb{P}$, or, if $\mathbb{P}$ is a set of ordered pairs, that $(X, Y) \in \mathbb{P}$ implies $(T(X), T(Y)) \in \mathbb{P}$.

An operator $T$ on $\mathbb{M}_{m, n}(\mathbb{S})$ is called a $(P, Q, B)$-operator if there exist permutation matrices $P \in \mathbb{M}_{m}(\mathbb{S})$ and $Q \in \mathbb{M}_{n}(\mathbb{S})$, and a matrix $B \in \mathbb{M}_{m, n}(\mathbb{S})$ with $B \geq J$ such that

$$
T(X)=P(X \circ B) Q
$$

for all $X \in \mathbb{M}_{m, n}(\mathbb{S})$ or, $m=n$ and

$$
T(X)=P(X \circ B)^{t} Q
$$

for all $X \in \mathbb{M}_{n}(\mathbb{S})$. Operators of the form (2.1) are called non-transposing $(P, Q, B)$-operators; operators of the form $(2.2)$ are transposing $(P, Q, B)$-operators.

An operator $T$ is called a $(U, V)$-operator if there exist invertible matrices $U \in \mathbb{M}_{m}(\mathbb{S})$ and $V \in \mathbb{M}_{n}(\mathbb{S})$ such that

$$
T(X)=U X V
$$

for all $X \in \mathbb{M}_{m, n}(\mathbb{S})$ or, $m=n$ and

$$
T(X)=U X^{t} V
$$

for all $X \in \mathbb{M}_{n}(\mathbb{S})$. Operators of the form (2.3) are called non-transposing $(U, V)$-operators; operators of the form (2.4) are transposing $(U, V)$-operators.

Lemma 2.9. Let $T$ be a $(P, Q, B)$-operator on $\mathbb{M}_{m, n}(\mathbb{S})$, where $m c(B)=1$ and all elements of $B$ are units in $\mathbb{Z}(\mathbb{S})$. If $\mathbb{S}$ is commutative, then $T$ is a $(U, V)$-operator.

Proof. Since $T$ is a $(P, Q, B)$-operator, so there exist permutation matrices $P \in$ $\mathbb{M}_{m}(\mathbb{S})$ and $Q \in \mathbb{M}_{n}(\mathbb{S})$ such that $T(X)=P(X \circ B) Q$, or $m=n$ and $T(X)=$ $P(X \circ B)^{t} Q$ for all $X \in \mathbb{M}_{m, n}(\mathbb{S})$. Since $m c(B)=1$, so it follows from (1.1) that $\operatorname{rank}(B)=1$, equivalently, there exist vectors $\mathbf{d}=\left(d_{1}, \ldots, d_{m}\right) \in \mathbb{S}^{m}$ and $\mathbf{e}=$ $\left(e_{1}, \ldots, e_{n}\right) \in \mathbb{S}^{n}$ such that $B=\mathbf{d}^{t} \mathbf{e}$. Since $b_{i, j}$ are units, $d_{i}$ and $e_{j}$ are invertible elements in $\mathbb{S}$ for all $(i, j) \in \Delta_{m, n}$. Let $D=\operatorname{diag}\left(d_{1}, \ldots, d_{m}\right) \in \mathbb{M}_{m}(\mathbb{S})$ and $E=\operatorname{diag}\left(e_{1}, \ldots, e_{n}\right) \in \mathbb{M}_{n}(\mathbb{S})$ be diagonal matrices. Since $\mathbb{S}$ is commutative, it is straightforward to check that $X \circ B=D X E$ for all $X \in \mathbb{M}_{m, n}(\mathbb{S})$. For the case of $T(X)=P(X \circ B) Q$, if we let $U=P D$ and $V=E Q$, then $T(X)=U X V$ for all $X \in \mathbb{M}_{m, n}(\mathbb{S})$. If $T$ is of the form $T(X)=P(X \circ B)^{t} Q$, then $U=P E$ and $V=D Q$ shows that $T(X)=U X^{t} V$ for all $X \in \mathbb{M}_{m, n}(\mathbb{S})$. Thus the Lemma follows.

If $A$ and $B$ are matrices and $A \geq B$ we let $A \backslash B$ denote the matrix $C=\left[c_{i, j}\right]$ where

$$
c_{i, j}=\left\{\begin{aligned}
0 & \text { if } b_{i, j} \neq 0 \\
a_{i, j} & \text { otherwise }
\end{aligned}\right.
$$


We recall some results proven in [2] for later use.

Theorem 2.10 ([2, Theorem 2.14]). Let $\mathbb{S}$ be an antinegative semiring without zero divisors and $T: \mathbb{M}_{m, n}(\mathbb{S}) \rightarrow \mathbb{M}_{m, n}(\mathbb{S})$ be a linear operator. Then the following are equivalent:

(1) $T$ is bijective.

(2) $T$ is surjective.

(3) There exists a permutation $\sigma$ on $\Delta_{m, n}$ and units $b_{i, j} \in \mathbb{Z}(\mathbb{S})$ such that $T\left(E_{i, j}\right)=b_{i, j} E_{\sigma(i, j)}$ for all $(i, j) \in \Delta_{m, n}$.

Lemma 2.11 ([2, Lemma 2.16]). Let $\mathbb{S}$ be an antinegative semiring without zero divisors, $T: \mathbb{M}_{m, n}(\mathbb{S}) \rightarrow \mathbb{M}_{m, n}(\mathbb{S})$ be an operator which maps lines to lines and is defined by $T\left(E_{i, j}\right)=b_{i, j} E_{\sigma(i, j)}$, where $\sigma$ is a permutation on $\Delta_{m, n}$, and $b_{i, j} \in \mathbb{Z}(\mathbb{S})$ are nonzero elements. Then $T$ is a $(P, Q, B)$-operator.

One can easily check that if $m=1$ or $n=1$, then all operators under consideration are $(P, Q, B)$-operators, if $m=n=1$, then all operators under consideration are $\left(P, P^{t}, B\right)$-operators.

Henceforth we will always assume that $m, n \geq 2$.

Now, we consider the following sets of matrices that arise as extremal cases in the inequalities listed in Theorem 2.5.

$$
\begin{aligned}
& \mathbb{M}_{1 L}(\mathbb{S})=\left\{(X, Y) \in \mathbb{M}_{n}(\mathbb{S})^{2} \mid m c(X Y)=m c(Y)\right\} \\
& \mathbb{M}_{2 N}(\mathbb{S})=\left\{(X, Y) \in \mathbb{M}_{n}(\mathbb{S})^{2} \mid m c(X Y)=0\right\} \\
& \mathbb{M}_{3 B}(\mathbb{S})=\left\{(X, Y) \in \mathbb{M}_{n}(\mathbb{S})^{2} \mid m c(X)+m c\left(Y^{t}\right)>n \text { and } m c(X Y)=1\right\} \\
& \mathbb{M}_{4 R}(\mathbb{S})=\left\{(X, Y) \in \mathbb{M}_{n}(\mathbb{S})^{2} \mid m c(X Y)=\rho(X)+\rho(Y)-n\right\} .
\end{aligned}
$$

In the following sections, we characterize the linear operators that preserve the sets $\mathbb{M}_{1 L}(\mathbb{S}), \mathbb{M}_{2 N}(\mathbb{S}), \mathbb{M}_{3 B}(\mathbb{S})$ and $\mathbb{M}_{4 R}(\mathbb{S})$.

\section{Linear operators that preserve $\mathbb{M}_{1 L}(\mathbb{S})$}

In this section, we investigate the linear operators that preserve the extreme set $\mathbb{M}_{1 L}(\mathbb{S})$. Recall that

$$
\mathbb{M}_{1 L}(\mathbb{S})=\left\{(X, Y) \in \mathbb{M}_{n}(\mathbb{S})^{2} \mid m c(X Y)=m c(Y)\right\} .
$$

Lemma 3.1. Let $T$ be a surjective linear operator on $\mathbb{M}_{n}(\mathbb{S})$ that preserves $\mathbb{M}_{1 L}(\mathbb{S})$. Then $T$ preserves lines.

Proof. Suppose that $T^{-1}$ does not map columns to lines, without loss of generality, that $T^{-1}\left(E_{1,1}+E_{2,1}\right) \geq E_{1,1}+E_{2,2}$. Then $T(I)$ has nonzero entries in at most $n-1$ columns. Suppose $T(I)$ has all zero entries in column $j$. Then for $X=I$ and $Y=T^{-1}\left(E_{j, 1}\right)$, we have $X Y=Y$ however, $T(X) T(Y)=O$. This contradicts the fact that $T$ preserves $\mathbb{M}_{1 L}(\mathbb{S})$.

Suppose that $T^{-1}$ does not map rows to lines, without loss of generality, that $T^{-1}\left(E_{1,1}+E_{1,2}\right) \geq E_{1,1}+E_{2,2}$. That is $T\left(E_{1,1}+E_{2,2}\right)=b_{1,1} E_{1,1}+b_{2,2} E_{1,2}$. 
Then for $X=b_{1,1}^{-1} E_{1,1}+b_{2,2}^{-1} E_{2,2}+\left[O_{2} \oplus I_{n-2}\right], T(X)$ has maximal column rank at most $n-1$ since either the first two columns of $T(X)$ are linearly dependent or at least one of the columns from the $3^{r d}$ through the $n^{\text {th }}$ is zero.

Let $Y=T^{-1}(I)$, then we have that $(X, Y) \in \mathbb{M}_{1 L}(\mathbb{S})$ since $m c(X Z)=$ $m c(Z)$ for any $Z$, while $m c(T(X) T(Y))=m c(T(X)) \leq n-1<n=m c(I)=$ $m c(T(Y))$ so that $(T(X), T(Y)) \notin \mathbb{M}_{1 L}(\mathbb{S})$, a contradiction.

Thus $T^{-1}$ and hence $T$ map lines to lines.

Theorem 3.2. Let $T$ be a surjective linear operator on $\mathbb{M}_{n}(\mathbb{S})$ that preserves $\mathbb{M}_{1 L}(\mathbb{S})$. Then $T$ is a non-transposing $\left(P, P^{t}, B\right)$-operator, where $m c(B)=1$ and all elements of $B$ are units in $\mathbb{Z}(\mathbb{S})$.

Proof. By applying Lemma 3.1 and Theorem 2.10 to Lemma 2.11, we have that if $T$ preserves $\mathbb{M}_{1 L}(\mathbb{S})$, then $T$ is a $(P, Q, B)$-operator.

Suppose that $m c(B) \geq 2$, without loss of generality $m c(B[1,2 \mid 1,2])=2$, and $E_{i, 1} Q P=E_{i, r}$ for all $i$. Consider the pair $X=E_{1,1}, Y=C_{1}+C_{2}$. Then $X Y=$ $E_{1,1}+E_{1,2}$ and $m c(X Y)=1=m c(Y)$. Thus $(X, Y) \in \mathbb{M}_{1 L}(\mathbb{S})$. However, the maximal column rank of $(X \circ B) Q P(Y \circ B))=b_{1, r} b_{r, 1} E_{1,1}+b_{1, r} b_{r, 2} E_{1,2}$ is 1 since $b_{1, r} b_{r, 1}=b_{r, 1} b_{r, 2}^{-1}\left(b_{1, r} b_{r, 2}\right)$ by assumption on $b_{i, j}\left(b_{i, j}\right.$ are units in $\mathbb{Z}(\mathbb{S}))$, that is, the columns of $(X \circ B) Q P(Y \circ B)$ are linearly dependent. Thus $m c(T(X) T(Y))=m c((X \circ B) Q P(Y \circ B))=1, m c(T(Y))=m c(Y \circ B) \geq$ $m c(B[1,2 \mid 1,2])=2$. Hence $(T(X), T(Y)) \notin \mathbb{M}_{1 L}(\mathbb{S})$, a contradiction. Thus $m c(B)=1$.

To see that the operator $T(X)=P(X \circ B)^{t} Q$ does not preserve $\mathbb{M}_{1 L}(\mathbb{S})$, it suffices to consider $T_{0}(X)=X^{t} D$, where $D=Q P$, since a similarity and a Hadamard product with a matrix of maximal column rank 1 and invertible entries preserve $\mathbb{M}_{1 L}(\mathbb{S})$. Let

$$
X=\left(D^{-1}\right)^{t}\left(\left[\begin{array}{cccc}
1 & 1 & 0 & 0 \\
1 & 1 & 0 & 0 \\
0 & 0 & 1 & 0 \\
0 & 0 & 0 & 1
\end{array}\right] \oplus I_{n-4}\right) \text { and } Y=\left[\begin{array}{cccc}
0 & 1 & 0 & 0 \\
0 & 0 & 0 & 0 \\
1 & 0 & 0 & 0 \\
1 & 1 & 0 & 0
\end{array}\right] \oplus I_{n-4}
$$

Then $(X, Y) \in \mathbb{M}_{1 L}(\mathbb{S})$ while $\left(X^{t} D, Y^{t} D\right) \notin \mathbb{M}_{1 L}(\mathbb{S})$. That is, the transposing operator does not preserve $\mathbb{M}_{1 L}(\mathbb{S})$.

It remains to prove that $Q=P^{t}$. Assume that $Q P \neq I$, and that $X \rightarrow$ $(Q P) X$ transforms the $r^{t h}$ row into the $t^{t h}$ row for some $r \neq t$. We consider the matrix $X=\sum_{i \neq t} E_{i, i}, Y=E_{r, r}$. Then $(X, Y) \in \mathbb{M}_{1 L}(\mathbb{S})$, while for certain invertible elements $b_{i, i} \in \mathbb{Z}(\mathbb{S})$ we have that $T(X) T(Y)=P(X \circ B) Q P(Y \circ$ $B) Q=P\left(\sum_{i \neq t} b_{i, i} E_{i, i}\right)\left(b_{r, r} E_{t, t}\right) Q=O$. Thus $(T(X), T(Y)) \notin \mathbb{M}_{1 L}(\mathbb{S})$, a contradiction.

Hence $Q=P^{t}$.

Corollary 3.3. Let $T$ be a surjective linear operator on $\mathbb{M}_{n}(\mathbb{S})$ with $n \geq 4$. If $\mathbb{S}$ is commutative and $1+1 \neq 1$, then $T$ preserves $\mathbb{M}_{1 L}(\mathbb{S})$ if and only if there exist 
an invertible matrix $U$ and an invertible element $\alpha$ such that $T(X)=\alpha U X U^{-1}$ for all $X \in \mathbb{M}_{n}(\mathbb{S})$.

Proof. Suppose $T$ preserves $\mathbb{M}_{1 L}(\mathbb{S})$. By Theorem 3.2, $T$ is a non-transposing $\left(P, P^{t}, B\right)$-operator, where $m c(B)=1$ and all elements of $B$ are units in $\mathbb{Z}(\mathbb{S})$. That is, $T(X)=P(X \circ B) P^{t}$ for all $X \in \mathbb{M}_{n}(\mathbb{S})$. In the proof of Lemma 2.9, there exist invertible diagonal matrices $D$ and $E$ in $\mathbb{M}_{n}(\mathbb{S})$ such that $X \circ B=$ $D X E$ and hence $T(X)=P D X E P^{t}$. Let us show that $E D$ is an invertible scalar matrix.

Define $L(X)=\left(E P^{t}\right) T(X)\left(E P^{t}\right)^{-1}$ for all $X \in \mathbb{M}_{n}(\mathbb{S})$. Then $L(X)=E D X$ for all $X \in \mathbb{M}_{n}(\mathbb{S})$. Since $T$ preserves $\mathbb{M}_{1 L}(\mathbb{S})$ if and only if $L$ does, it suffices to consider $L(X)=E D X$. Let $G=E D$. Then $G=\operatorname{diag}\left(g_{1}, \ldots, g_{n}\right)$ is an invertible diagonal matrix. Assume that $g_{1} \neq g_{2}$. Consider matrices

$$
A=\left[\begin{array}{llll}
0 & 4 & 1 & 1 \\
4 & 0 & 1 & 1 \\
1 & 1 & 0 & 1 \\
1 & 1 & 1 & 0
\end{array}\right] \text { and } B=\left[\begin{array}{cccc}
1 & 1 & 0 & 0 \\
1 & 1 & 0 & 0 \\
0 & 0 & 1 & 1 \\
0 & 0 & 1 & 1
\end{array}\right]
$$

Let $X=A \oplus O_{n-4}$ and $Y=G^{-1}\left(B \oplus O_{n-4}\right)$. Since all columns of $A$ are linearly independent, it follows that $m c(A)=m c(X)=m c(L(X))=4$ and $m c(B)=$ $m c(Y)=m c(L(Y))=2$. Furthermore,

$$
X Y=\left[\begin{array}{cccc}
4 g_{2}^{-1} & 4 g_{2}^{-1} & g_{3}^{-1}+g_{4}^{-1} & g_{3}^{-1}+g_{4}^{-1} \\
4 g_{1}^{-1} & 4 g_{1}^{-1} & g_{3}^{-1}+g_{4}^{-1} & g_{3}^{-1}+g_{4}^{-1} \\
g_{1}^{-1}+g_{2}^{-1} & g_{1}^{-1}+g_{2}^{-1} & g_{4}^{-1} & g_{4}^{-1} \\
g_{1}^{-1}+g_{2}^{-1} & g_{1}^{-1}+g_{2}^{-1} & g_{3}^{-1} & g_{3}^{-1}
\end{array}\right] \oplus O_{n-4}
$$

has maximal column rank at most 2. If $m c(X Y)=1$, then we can easily show that $g_{1}=g_{2}$, a contradiction. Thus $m c(X Y)=2$. That is $(X, Y) \in \mathbb{M}_{1 L}(\mathbb{S})$. But $L(X) L(Y)=G\left(\left[\begin{array}{llll}4 & 4 & 2 & 2 \\ 4 & 4 & 2 & 2 \\ 2 & 2 & 1 & 1 \\ 2 & 2 & 1 & 1\end{array}\right] \oplus O_{n-4}\right)$ has maximal column rank 1 and hence $(L(X), L(Y)) \notin \mathbb{M}_{1 L}(\mathbb{S})$. This contradiction shows that $g_{1}=g_{2}$. Similarly, if we consider a matrix $A^{\prime}=\left[\begin{array}{llll}0 & 1 & 1 & 1 \\ 1 & 0 & 1 & 1 \\ 1 & 1 & 0 & 4 \\ 1 & 1 & 4 & 0\end{array}\right]$, then the parallel argument shows that $g_{3}=g_{4}$. Generally, if $n \geq 5$, then we can split zero block into two parts and take $X^{\prime}=O_{r} \oplus A \oplus O_{n-r-4}$ or $X^{\prime}=O_{r} \oplus A^{\prime} \oplus O_{n-r-4}$ for appropriate $r$. Therefore we have that $G$ is an invertible scalar matrix. That is, $G=E D=\alpha I$ for some invertible element $\alpha$, equivalently $E=\alpha D^{-1}$. If we let $U=P D$, then $T(X)=P(D X E) P^{t}=\alpha(P D) X(P D)^{-1}=\alpha U X U^{-1}$ for all $X \in \mathbb{M}_{n}(\mathbb{S})$. Thus the result follows.

The converse is obvious from the construction of the linear operator $T$. 


\section{Linear operators that preserve $\mathbb{M}_{2 N}(\mathbb{S})$}

In this section, we investigate the linear operators that preserve the extreme set $\mathbb{M}_{2 N}(\mathbb{S})$. Recall that

$$
\mathbb{M}_{2 N}(\mathbb{S})=\left\{(X, Y) \in \mathbb{M}_{n}(\mathbb{S})^{2} \mid m c(X Y)=0\right\} .
$$

Now, observe that $m c(A)=0$ if and only if $A=0$ if and only if $\operatorname{rank}(A)=0$. Thus by $[2$, Corollary 7.2] we have a characterization of the linear operator that preserve $\mathbb{M}_{2 N}(\mathbb{S})$ as follows:

Theorem 4.1. Let $T$ be a surjective linear operator on $\mathbb{M}_{n}(\mathbb{S})$. Then $T$ preserves $\mathbb{M}_{2 N}(\mathbb{S})$ if and only if $T$ is a non-transposing $\left(P, P^{t}, B\right)$-operator, where all elements of $B$ are units in $\mathbb{Z}(\mathbb{S})$.

\section{Linear operators that preserve $\mathbb{M}_{3 B}(\mathbb{S})$}

In this section, we investigate the linear operators that preserve the extreme set $\mathbb{M}_{3 B}(\mathbb{S})$. Recall that

$$
\mathbb{M}_{3 B}(\mathbb{S})=\left\{(X, Y) \in \mathbb{M}_{n}(\mathbb{S})^{2} \mid m c(X)+m c\left(Y^{t}\right)>n \text { and } m c(X Y)=1\right\} .
$$

Lemma 5.1. Let $T$ be a surjective linear operator on $\mathbb{M}_{n}(\mathbb{S})$. If $T$ preserves $\mathbb{M}_{3 B}(\mathbb{S})$, then $T$ preserves lines.

Proof. Recall that if $(X, Y) \in \mathbb{M}_{3 B}(\mathbb{S})$, then $m c(X)+m c\left(Y^{t}\right)>n$. We assume that $T$ does not preserve lines. Then there exist indices $i, j, k, l, i \neq k, j \neq l$ such that nonzero entries of $T\left(E_{i, j}\right)$ and $T\left(E_{k, l}\right)$ lie in a line.

Let $T\left(E_{i, j}\right)=b_{i, j} E_{s, t}$. Then either $T\left(E_{k, l}\right)=b_{k, l} E_{s, t^{\prime}}$ or $T\left(E_{k, l}\right)=b_{k, l} E_{s^{\prime}, t}$. In both cases $m c\left(T\left(E_{i, j}+E_{k, l}\right)\right)=1$. Let $Y^{\prime} \in \mathbb{M}_{n}(\mathbb{S})$ be a matrix such that $Y^{\prime}+E_{j, i}+E_{l, k}$ is a permutation matrix. We consider $X=E_{i, j}+E_{k, l}$, $Y=Y^{\prime}+E_{l, k}$. Then $X Y=E_{k, k}$ and $(X, Y) \in \mathbb{M}_{3 B}(\mathbb{S})$. However, since $m c(T(X))=1$ in either case, and $m c\left(T(Y)^{t}\right) \leq n-1, m c(T(X))+m c\left(T(Y)^{t}\right) \leq$ $n$. Therefore, we have that $(T(X), T(Y)) \notin \mathbb{M}_{3 B}(\mathbb{S})$, a contradiction.

Hence $T$ preserves lines.

Theorem 5.2. Let $n \geq 3$ and $T$ be a surjective linear operator on $\mathbb{M}_{n}(\mathbb{S})$ that preserves $\mathbb{M}_{3 B}(\mathbb{S})$. Then $T$ is a non-transposing $\left(P, P^{t}, B\right)$-operator, where $m c(B)=1$ and all elements of $B$ are units in $\mathbb{Z}(\mathbb{S})$.

Proof. By applying Lemma 5.1 and Theorem 2.10 to Lemma 2.11, we have that if $T$ preserves $\mathbb{M}_{3 B}(\mathbb{S})$, then $T$ is a $(P, Q, B)$-operator.

Suppose that $m c(B) \geq 2$, without loss of generality $m c(B[1,2 \mid 1,2])=2$, and $E_{i, 1} Q P=E_{i, r}, E_{i, 2} Q P=E_{i, s}$ for all $i$. Consider the pair $X=C_{1}+C_{2}$, $Y=I$. Then $(X, Y) \in \mathbb{M}_{3 B}(\mathbb{S})$ while $(T(X), T(Y)) \notin \mathbb{M}_{3 B}(\mathbb{S})$, a contradiction. Thus $m c(B)=1$.

To see that the operator $T(X)=P(X \circ B)^{t} Q$ does not preserve $\mathbb{M}_{3 B}(\mathbb{S})$, it suffices to consider $T_{0}(X)=X^{t} D$, where $D=Q P$, since a similarity and a Hadamard product with a matrix of maximal column rank 1 and invertible entries preserve $\mathbb{M}_{3 B}(\mathbb{S})$. 
Let $X=\left(D^{-1}\right)^{t}\left[\begin{array}{ll}O & I_{2} \\ O & O\end{array}\right]$ and $Y=\left[\begin{array}{cc}I_{n-1} & O \\ O & O\end{array}\right]$. Then $(X, Y) \in \mathbb{M}_{3 B}(\mathbb{S})$ while $\left(X^{t} D, Y^{t} D\right) \notin \mathbb{M}_{3 B}(\mathbb{S})$. This proves that $T$ is a non-transposing $(P, Q, B)$ operator.

Let us check that $Q=P^{t}$. Assume that $Q P \neq I$, and that $X \rightarrow(Q P) X$ transforms the $p^{t h}$ row into the $s^{\text {th }}$ row and $r^{\text {th }}$ row into $t^{\text {th }}$ row with $r \neq s, t$. These exist since $n \geq 3$. We consider the matrix $X=\sum_{i \neq r} E_{i, i}, Y=E_{p, p}+$ $E_{r, r}$. Then $(X, Y) \in \mathbb{M}_{3 B}(\mathbb{S})$. And we have that $m c(T(X))+m c(T(Y))=$ $n+1>n$ and $T(X) T(Y)=P(X \circ B) Q P(Y \circ B) Q=P\left(\sum_{i \neq r} b_{i, i} E_{i, i}\right)\left(b_{p, p} E_{s, p}+\right.$ $\left.b_{r, r} E_{t, s}\right) Q$. Thus $m c\left((T(X) T(Y))=2\right.$, that is, $(T(X), T(Y)) \notin \mathbb{M}_{3 B}(\mathbb{S})$, a contradiction.

Hence $Q=P^{t}$.

Corollary 5.3. Let $\mathbb{S}=\mathbb{B}, \mathbb{Z}^{+}$or a chain semiring, and $T$ be a surjective linear operator on $\mathbb{M}_{n}(\mathbb{S})$ with $n \geq 3$. Then $T$ preserves $\mathbb{M}_{3 B}(\mathbb{S})$ if and only if there is a permutation matrix $P \in \mathbb{M}_{n}(\mathbb{S})$ such that $T(X)=P X P^{t}$ for all $X \in \mathbb{M}_{n}(\mathbb{S})$.

Proof. Suppose $T$ preserves $\mathbb{M}_{3 B}(\mathbb{S})$. By Theorem 5.2, $T$ is a non-transposing $\left(P, P^{t}, B\right)$-operator, where all elements of $B$ are invertible. Note that if $\mathbb{S}=$ $\mathbb{B}, \mathbb{Z}^{+}$or a chain semiring, " 1 " is the only invertible element in $\mathbb{S}$, and hence $B=J$. Thus, there exists a permutation matrix $P \in \mathbb{M}_{n}(\mathbb{S})$ such that $T(X)=$ $P X P^{t}$ for all $X \in \mathbb{M}_{n}(\mathbb{S})$.

The converse is easily established.

\section{Linear operators that preserve $\mathbb{M}_{4 R}(\mathbb{S})$}

In this section, we investigate the linear operators that preserve the extreme set $\mathbb{M}_{4 R}(\mathbb{S})$. Recall that

$$
\mathbb{M}_{4 R}(\mathbb{S})=\left\{(X, Y) \in \mathbb{M}_{n}(\mathbb{S})^{2} \mid m c(X Y)=\rho(X)+\rho(Y)-n\right\} .
$$

Lemma 6.1. Let $\mathbb{S}$ be any subsemiring of $\mathbb{R}^{+}, \sigma$ be a permutation of $\Delta_{n}$, and $T$ be defined by $T\left(E_{i, j}\right)=b_{i, j} E_{\sigma(i, j)}$ for all $(i, j) \in \Delta_{n}$, where all $b_{i, j}$ are units. If $T$ preserves $\mathbb{M}_{4 R}(\mathbb{S})$, then $T$ preserves lines.

Proof. If $T$ does not preserve lines, then there exist indices $i, j, k, l, i \neq k, j \neq l$ such that nonzero entries of $T\left(E_{i, j}\right)$ and $T\left(E_{k, l}\right)$ lie in a line. Let $X^{\prime} \in \mathbb{M}_{n}(\mathbb{S})$ be a matrix such that $X^{\prime}+E_{i, j}+E_{k, l}$ is a permutation matrix.

We consider $X=X^{\prime}+E_{i, j}+E_{k, l}$. Then $(X, O) \in \mathbb{M}_{4 R}(\mathbb{S})$. However, $m c(T(X)) \leq n-1, \rho(T(X)) \leq n-1$ since either $T(X)$ has a zero column or $T(X)$ has two proportional columns since $b_{i, j}$ is invertible. Thus $(T(X), O) \notin$ $\mathbb{M}_{4 R}(\mathbb{S})$, a contradiction.

Hence $T$ preserves lines.

Theorem 6.2. Let $\mathbb{S}$ be a subsemiring of $\mathbb{R}^{+}$, and $T$ be a surjective linear operator on $\mathbb{M}_{n}(\mathbb{S})$. If $T$ preserves $\mathbb{M}_{4 R}(\mathbb{S})$, then $T$ is a non-transposing $\left(P, P^{t}, B\right)$ operator, where $m c(B)=1$ and all elements of $B$ are units. 
Proof. By applying Lemma 6.1 and Theorem 2.10 to Lemma 2.11, we have that if $T$ preserves $\mathbb{M}_{4 R}(\mathbb{S})$, then $T$ is a $(P, Q, B)$-operator.

Let us check that $Q=P^{t}$. Assume that $Q P \neq I$, and that $X \rightarrow(Q P) X$ transforms the $r^{\text {th }}$ row into the $t^{\text {th }}$ row with $r \neq t$. We consider the matrix $X=\sum_{i \neq r} E_{i, i}, Y=E_{r, r}$. Then $(X, Y) \in \mathbb{M}_{4 R}(\mathbb{S})$, and for certain nonzero $b_{i, i} \in \mathbb{S}, T(X) T(Y)=P(X \circ B) Q P(Y \circ B) Q=P\left(\sum_{i \neq r} b_{i, i} E_{i, i}\right)\left(b_{r, r} E_{t, r}\right) Q \neq O$, that is, $(T(X), T(Y)) \notin \mathbb{M}_{4 R}(\mathbb{S})$, a contradiction. Thus $Q=P^{t}$. Let

Suppose that $m c(B) \geq 2$, without loss of generality $m c(B[1,2 \mid 1,2])=2$.

$$
Y=\left[\begin{array}{ccc}
b_{1,1}^{-1} & b_{1,2}^{-1} & 0 \\
b_{2,1}^{-1} & b_{2,2}^{-1} & 0 \\
O & & I_{n-2}
\end{array}\right]
$$

Then $m c(Y)=m c\left(Y^{2}\right)=n$. Note that from the invertibility of $b_{i, j}$ it follows that $\rho(Y)=n$. Indeed, if $b_{i, 1}^{-1}=\lambda b_{i, 2}^{-1}(i=1,2)$ for some $\lambda \in \mathbb{R}^{+}$, then $\lambda=$ $b_{i, 1}^{-1} b_{i, 2} \in \mathbb{S}$ which contradicts $m c(B[1,2 \mid 1,2])=2$. Thus $m c\left(Y^{2}\right)=2 \rho(Y)-n$ or $(Y, Y) \in \mathbb{M}_{4 R}(\mathbb{S})$. But $m c(Y \circ B)=m c\left((Y \circ B)^{2}\right)=\rho(Y \circ B)=n-1$, and hence $m c\left((Y \circ B)^{2}\right)>2 \rho(Y \circ B)-n$, so that $(T(X), O) \notin \mathbb{M}_{4 R}(\mathbb{S})$, a contradiction. Hence $m c(B)=1$.

Let $X=\left(D^{-1}\right)^{t}\left(\left[\begin{array}{ll}0 & 1 \\ 1 & 1\end{array}\right] \oplus I_{n-2}\right), Y=\left[\begin{array}{ll}1 & 0 \\ 0 & 0\end{array}\right] \oplus I_{n-2}$, where $D=Q P$. Then $(X, Y) \in \mathbb{M}_{4 R}(\mathbb{S})$ while $\left(X^{t} D, Y^{t} D\right) \notin \mathbb{M}_{4 R}(\mathbb{S})$. This proves that $T$ is a nontransposing $(P, Q, B)$-operator.

Therefore $T$ is a non-transposing $\left(P, P^{t}, B\right)$-operator, where $m c(B)=1$.

Corollary 6.3. Let $\mathbb{S}$ be a subsemiring of $\mathbb{R}^{+}$, and $T$ be a surjective linear operator on $\mathbb{M}_{n}(\mathbb{S})$, where $n \geq 4$. Then $T$ preserves $\mathbb{M}_{4 R}(\mathbb{S})$ if and only if there is an invertible matrix $U$ and an invertible elements $\alpha$ such that $T(X)=$ $\alpha U X U^{-1}$ for all $X \in \mathbb{M}_{n}(\mathbb{S})$.

Proof. Suppose $T$ preserves $\mathbb{M}_{4 R}(\mathbb{S})$. By Theorem $6.2, T$ is a non-transposing $\left(P, P^{t}, B\right)$-operator, where $m c(B)=1$ and all elements of $B$ are units; $T(X)=$ $P(X \circ B) P^{t}$ for all $X \in \mathbb{M}_{n}(\mathbb{S})$. In the proof of Lemma 2.9 , there exist invertible diagonal matrices $D$ and $E$ in $\mathbb{M}_{n}(\mathbb{S})$ such that $X \circ B=D X E$ and hence that $T(X)=P D X E P^{t}$. Let us show that $E D$ is an invertible scalar matrix. Similar to the proof of Corollary 3.3, we suffice to consider $L(X)=E D X$ for all $X \in \mathbb{M}_{n}(\mathbb{S})$. Let $G=E D$. Then $G=\operatorname{diag}\left(g_{1}, \ldots, g_{n}\right)$ is an invertible diagonal matrix. Suppose $G$ is not a scalar matrix. As in Corollary 3.3, we lose no generality in assuming that $g_{1} \neq g_{2}$. Let $A$ and $B$ be matrices in (3.1). Let $X=A \oplus I_{n-4}$ and $Y=B \oplus I_{n-4}$. Then

$$
X Y=\left[\begin{array}{llll}
4 & 4 & 2 & 2 \\
4 & 4 & 2 & 2 \\
2 & 2 & 1 & 1 \\
2 & 2 & 1 & 1
\end{array}\right] \oplus O_{n-4}
$$


so that $\rho(X)=n-1=\rho(L(X)), \rho(Y)=n-2=\rho(T(Y))$, and $m c(X Y)=n-3$. Thus $(X, Y) \in \mathbb{M}_{4 R}(\mathbb{S})$. But

$$
L(X) L(Y)=G\left(\left[\begin{array}{cccc}
4 g_{2} & 4 g_{2} & g_{3}+g_{4} & g_{3}+g_{4} \\
4 g_{1} & 4 g_{1} & g_{3}+g_{4} & g_{3}+g_{4} \\
g_{1}+g_{2} & g_{1}+g_{2} & g_{4} & g_{4} \\
g_{1}+g_{2} & g_{1}+g_{2} & g_{3} & g_{3}
\end{array}\right] \oplus I_{n-4}\right)
$$

so that $m c(L(X) L(X))=n-2$ because $g_{1} \neq g_{2}$. Thus $(L(X), L(Y)) \notin \mathbb{M}_{4 R}(\mathbb{S})$, a contradiction. Hence $G=E D=\alpha I$ for some invertible element $\alpha$. If $U=P D$, then $T(X)=\alpha U X U^{-1}$.

The converse is immediate.

\section{References}

[1] L. B. Beasley and A. E. Guterman, Rank inequalities over semirings, J. Korean Math. Soc. 42 (2005), no. 2, 223-241.

[2] L L - Lroblems for rank inequalities over semirings: factorization rank, Sovrem. Mat. Prilozh. No. 13, Algebra (2004), 53-70; translation in J. Math. Sci. (N. Y.) 131 (2005), no. 5, 5919-5938.

[3] L. B. Beasley, S.-G. Lee, and S.-Z. Song, Linear operators that preserve pairs of matrices which satisfy extreme rank properties, Linear Algebra Appl. 350 (2002), 263-272.

[4] S.-G. Hwang, S.-J. Kim, and S.-Z. Song, Linear operators that preserve maximal column rank of Boolean matrices, Linear and Multilinear Algebra 36 (1994), no. 4, 305-313.

[5] S. Pierce and others, A survey of linear preserver problems, Linear and Multilinear Algebra 33 (1992), no. 1-2. Gordon and Breach Science Publishers, Yverdon, 1992. pp. $1-129$.

[6] S.-Z. Song, Linear operators that preserve maximal column ranks of nonnegative integer matrices, Proc. Amer. Math. Soc. 126 (1998), no. 8, 2205-2211.

SEOK-Zun Song

Department of Mathematics

Jeju National University

JEJU 690-756, KoREA

E-mail address: szsong@cheju.ac.kr

KWON-RYONG PARK

Department of Mathematics

Jeju National University

JEJU 690-756, KOREA

E-mail address: aljo1004@naver.com

L. Hernandez Encinas

Department of Information Processing and Coding

Applied Physics Institute, CSIC

C/Serrano 144, E-28006 Madrid, Spain

E-mail address: luis@iec.csic.es 